\title{
How skyrmion lattice forms and arranges
}

Ping Huang ${ }^{1}$, Rajeswari Jayaraman ${ }^{2}$, Yoshie Murooka ${ }^{2}$, Marco Cantoni ${ }^{3}$, Arnaud Magrez ${ }^{4}$, Fabrizio Carbone ${ }^{2}$, Henrik Ronnow ${ }^{1}$ ${ }^{1}$ Laboratory For Quantum Magnetism, EPFL, Lausanne, Switzerland, ${ }^{2}$ Laboratory for ultrafast microscopy and electron scattering, EPFL, Lausanne, Switzerland, ${ }^{3}$ Interdisciplinary Centre for Electron Microscopy, EPFL, Lausanne, Switzerland, ${ }^{4}$ Crystal Growth Facility, EPFL, Lausanne, Switzerland E-mail: ping.huang@epfl.ch

Magnetic skyrmions, as topological spin textures, have been attracting rapidly increasing attention due to its highly possible application in spintronics. Besides appearing at the interfaces of thin layers of heavy metals and ferromagnets, where skyrmions are thought to be non-interacting localized spin textures, lattices of skyrmions are found in B20 type compounds such as $\mathrm{MnSi}, \mathrm{FeGe}$ and Cu2OSeO3. While small angle neutron scattering demonstrates the existence of such two dimensional hexagonal lattice, little was known about the formation and arrangement of the skyrmion lattice (SkL). Here, we will talk about our systematic real space investigation of the SkL by Lorentz Transmission Electron Microscopy (LTEM) to address this topic.

Phase transition from the helical phase to the skyrmion phase induced by magnetic field was observed directly in a nano-slab Cu2OSeO3 sample. It is a first order phase transition and is characterized by a glassy co-existence state of the both magnetic textures. Various defects can be seen, in both the helical phase and the skyrmion phase, upon thermal excitation. Dislocations, as a type of topological defect, are paid special attention in our work since it can affect the order of the SkL in terms of both short range and long range. Our results and analysis provide deep understanding of the thermodynamics of the SkL.

[1] Jayaraman R. et al. (2015), Proc.Natl. Acad. of Sci., 112, 14212.

Keywords: skyrmion lattice, phase transition, dislocation 